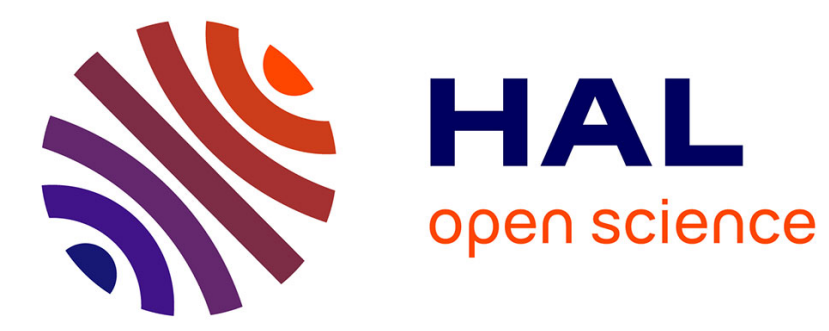

\title{
Terahertz responsivity of field effect transistors versus their static channel conductivity and loading effects
}

\author{
M. Sakowicz, M. B. Lifshits, O. A. Klimenko, F. Schuster, D. Coquillat, F.
}

Teppe, W. Knap

\section{- To cite this version:}

M. Sakowicz, M. B. Lifshits, O. A. Klimenko, F. Schuster, D. Coquillat, et al.. Terahertz responsivity of field effect transistors versus their static channel conductivity and loading effects. Journal of Applied Physics, 2011, 110, pp.4512. 10.1063/1.3632058 . hal-00635960

\section{HAL Id: hal-00635960 \\ https://hal.science/hal-00635960}

Submitted on 26 Oct 2011

HAL is a multi-disciplinary open access archive for the deposit and dissemination of scientific research documents, whether they are published or not. The documents may come from teaching and research institutions in France or abroad, or from public or private research centers.
L'archive ouverte pluridisciplinaire HAL, est destinée au dépôt et à la diffusion de documents scientifiques de niveau recherche, publiés ou non, émanant des établissements d'enseignement et de recherche français ou étrangers, des laboratoires publics ou privés. 


\title{
Terahertz responsivity of field effect transistors versus their static channel conductivity and loading effects
}

\author{
M. Sakowicz, ${ }^{1, a)}$ M. B. Lifshits, ${ }^{1,2}$ O. A. Klimenko, ${ }^{1,3}$ F. Schuster, ${ }^{1,4}$ D. Coquillat, ${ }^{1}$ F. Teppe, ${ }^{1}$ \\ and W. Knap ${ }^{1}$ \\ ${ }^{1}$ Laboratoire Charles Coulomb UMR 5221 and TERALAB, Université Montpellier2 and CNRS, \\ Montpellier 34090, France \\ ${ }^{2}$ A.F. Ioffe Physico-Technical Institute, St-Petersburg 194021, Russia \\ ${ }^{3}$ P.N. Lebedev Physical Institute of RAS, 53 Leninskiy Prospect, Moscow 119991, Russia \\ ${ }^{4}$ CEA-LETI, MINATEC Campus, 17 rue des Martyrs, Grenoble 38054, France
}

(Received 12 May 2011; accepted 29 July 2011; published online 12 September 2011)

\begin{abstract}
We study the broadband photovoltaic response of field effect transistors on terahertz radiation. A simple physical analytical model of the response is developed. It is based on plasma density perturbation in the transistor channel by the incoming terahertz radiation. The model shows how the non-resonant detection signal is related to static $(d c)$ transistor characteristics. We analyze loading effects related to capacitive, inductive, and resistive coupling of the detector to the read-out circuit as a function of modulation frequencies and loading resistors. As we show, the proposed physical model completed by loading effects fully describes the experimental results on the non-resonant sub-terahertz detection by all studied III-V (GaAs, GaN) and silicon based transistors. Field effect transistors were recently proposed as the best terahertz detecting pixels for fabrication of low cost focal plane arrays for terahertz imaging. This article gives prospects for electrical simulation of these transistors and their optimal integration in the focal plane arrays. (C) 2011 American Institute of Physics. [doi:10.1063/1.3632058]
\end{abstract}

\section{INTRODUCTION}

Field effect transistors (FETs) were found to be efficient terahertz (THz) detectors. ${ }^{1-12}$ The phenomena can be described using the Dyakonov-Shur theory ${ }^{11,13,14}$ in terms of the excitation of the 2-dimensional electron plasma in the transistor channel by the incoming $\mathrm{THz}$ radiation. When a $\mathrm{THz}$ field is applied between the gate and the source terminal of the transistor, it modulates at the same time the carrier velocity and the carrier density. This leads to the nonlinearity. $\mathrm{THz}$ electric field is rectified like in square law detectors and a constant $(d c)$ source-to-drain voltage appears. This voltage is the measured detection signal and is called the photovoltaic response or the photoresponse. More details about the detection mechanism can be found in a review-Ref. 11. Different kinds of FETs, like GaAs high electron mobility transistors (HEMTs), ${ }^{1,4}$ GaN HEMTs, ${ }^{4,10}$ InGaAs HEMTs, ${ }^{2}$ or silicon metal-oxide-semiconductor-FETs (MOSFETs), ${ }^{6,8}$ with a gate length of an order of hundreds of nanometers exhibit good broadband responsivities for the $\mathrm{THz}$ radiation. From the point of view of applications, the silicon MOSFETs are the most important ones due to low production costs and the ease of integration in electrical circuits. Recently, the first imagining experiments with GaAs FETs (Ref. 15) and with focal plane arrays made in silicon complementary metal-oxidesemiconductor (CMOS) technology ${ }^{16-18}$ were reported.

A standard method of investigation of $\mathrm{THz}$ detection by FETs is the measurement of the photoresponse versus the

\footnotetext{
${ }^{a)}$ Electronic mail: maciej.sakowicz@umontreal.ca. Present address: Département de Physique, Université de Montréal, C.P. 6128, Succ. Centre-ville, Montréal (QC) H3C 3J7, Canada.
}

gate voltage. Basic detection theory ${ }^{14}$ predicts divergence of the photoresponse signal when the gate voltage approaches the threshold voltage, whereas all experiments show a signal drop in the subthreshold region. This discrepancy comes from the assumption of linear dependence of the carrier density on the gate voltage in the Dyakonov-Shur theory, which does not hold in the subthreshold region. Taking into account the exponential dependence, in this region, the photoresponse tends to a constant value rather than diverges. ${ }^{4}$ In most of the experiments, however, one observes the signal drop in the subthreshold region. This drop observed in the leaky GaAs HEMTs was initially associated with the gate leakage current. ${ }^{4}$ Later the phenomenon was also observed in the very low leakage $\mathrm{Si}-\mathrm{CMOS}$ transistors and was explained as an effect related to the impedance mismatch between the detector and the external measurement (read out) systemloading effects. ${ }^{16,19}$ Even though the reasons for the signal drop were given, there was no simple way to predict and simulate accurately the experimental data. The presented models ${ }^{4,19}$ describe the real shape of the detection signal rather poorly. This is because of two main reasons: (i) the full impedance, capacitance in particular, of the read-out circuit was not taken into account ${ }^{16,19}$ and (ii) the gate voltage dependence of the carrier mobility was not taken into account.

In this work, we present an extensive experimental study of the non-resonant $\mathrm{THz}$ response of various high quality (low gate leakage current) III-V (GaAs, GaN) and silicon based FETs. We formulate a physical model which describes a relation between the non-resonant detection and the transistor channel $d c$ conductivity. It allows for analytical calculation of the expected $\mathrm{THz}$ photoresponse using static $(d c)$ 
TABLE I. The main parameters of the transistors.

\begin{tabular}{lcccc}
\hline \hline Transistor type & $L_{g}(\mu \mathrm{m})$ & $W_{g}(\mu \mathrm{m})$ & $m_{\text {eff }} / m_{e}$ & $\mu_{0}\left(\mathrm{~cm}^{2} /(\mathrm{Vs})\right)$ \\
\hline GaN HEMT (Ref. 20) & 0.25 & 100 & 0.2 & 1500 \\
GaAs HEMT (Ref. 21) & 0.15 & 50 & 0.067 & 8500 \\
Si MOSFET N1, CMOS 130 nm & 0.13 & 0.13 & 0.19 & 100 \\
Si MOSFET N2, CMOS 130 nm & 0.13 & 0.25 & 0.19 & 100 \\
(Ref. 22) & & & & \\
\hline \hline
\end{tabular}

transfer characteristics of a FET. We show that in the case of low gate leakage transistors, the presented physical model completed by taking into account resistance and capacitance loading effects can fully describe the $\mathrm{THz}$ photoresponse for all investigated III-V (GaAs, GaN) and silicon based FETs.

\section{EXPERIMENTAL DETAILS}

The goal of our experiments was to find a relation between the broadband $\mathrm{THz}$ photoresponse and the $d c$ channel conductivity of a FET and to understand the influence of geometric, material, and electronic parameters on it. We studied three types of FETs made of different materials and using different technologies-GaN and GaAs HEMTs and $\mathrm{Si}$ MOSFETs. Table I gathers the main parameters of the transistors, such as the gate length, $L_{g}$, and the gate width, $W_{g}$, the electron effective mass in the channel, $m_{e f f}$, and the room temperature electron mobility, $\mu_{0}$. For all the transistors we measured the dependence of the photoresponse on the gate voltage together with their $d c$ transfer characteristics.

Most of the experiments were done at room temperature but we collected data at some lower temperatures too. To cool down the transistors we used a continuous flow cryostat operating in the range between $150 \mathrm{~K}$ and $300 \mathrm{~K}$. An optical system based on parabolic mirrors was used to focus the radiation beam onto the samples. The photoresponse was measured by a standard lock-in technique using an external preamplifier $(25 \times)$ with an input resistance of $10 \mathrm{M} \Omega$. In each measurement session, the capacitance of the read-out circuit together with cables connecting the transistor to the preamplifier was determined by RLC measuring bridge. Typical value of the capacitance obtained was of the order of $150 \mathrm{pF}$. A backward wave oscillator operating at $0.23 \mathrm{THz}$ and a 0.3 $\mathrm{THz}$ electronic multiplier based $\mathrm{THz}$ source were used. The intensity of the incident radiation was modulated either by a mechanical chopper or by an internal electronic modulation of the backward wave oscillator. We measured the photoresponse as a function of the gate voltage, $V_{g}$, for modulation frequencies, $\omega_{m}$, ranging from $23 \mathrm{~Hz}$ to $10 \mathrm{kHz}$, and for different values of external load resistors, $R_{L}$ (see Fig. 1).

\section{MODEL}

We present a simple physical model relating the transistor photoresponse with its $d c$ channel conductivity. The Dyakonov-Shur theory ${ }^{14}$ was originally developed under the assumption that the mobility in the channel does not depend on the gate-to-channel voltage, $U$. However, this is not true in general. Usually it is difficult to determine simultaneously both the carrier density and the carrier mobility as functions

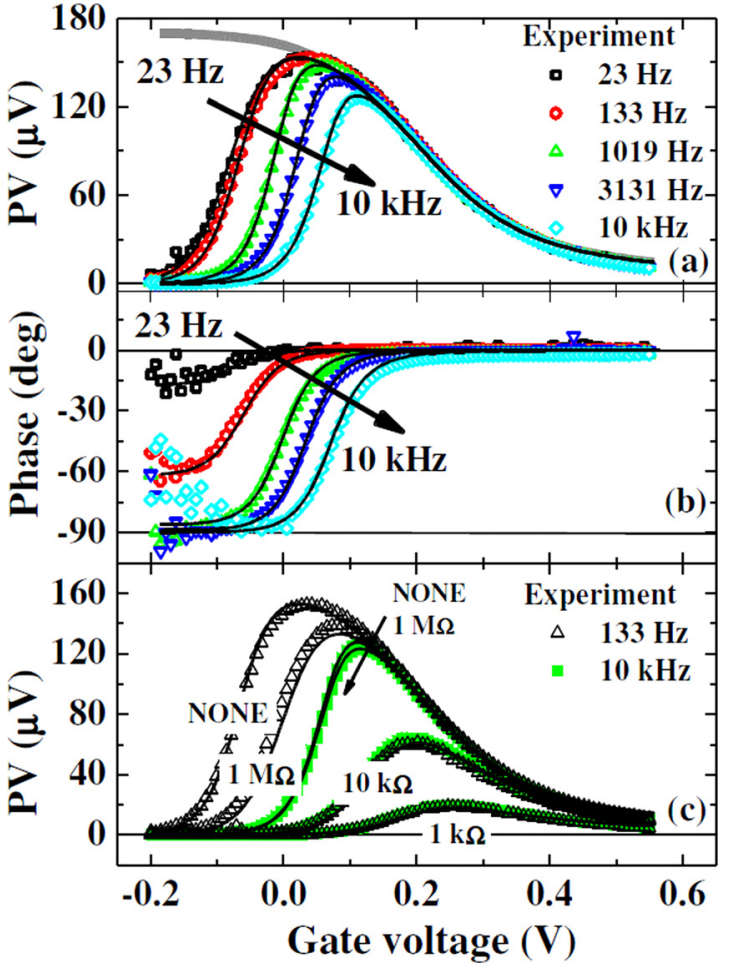

FIG. 1. (Color online) Si MOSFET N1: Measured magnitude (a) and (c) and phase (b) of the photovoltage (points) for different radiation modulation frequencies (a) and (b) and different external load resistors (c) together with model curves calculated from Eq. (15) (thin black lines) with the parameter $A=1.25 \times 10^{-4} \mathrm{~V}^{2}$ and the detection limit calculated from Eq. (14) (thick gray line, part (a)).

of the gate voltage. On the other hand, we can easily find the channel conductivity, $\sigma$, from the $d c$ transfer characteristics of a FET. Below we show that in case of a non-resonant detection, the knowledge of the $\sigma\left(V_{g}\right)$ dependence itself is sufficient to determine the source-to-drain photovoltage, $\Delta U$, induced by the $\mathrm{THz}$ radiation as a function of $V_{g}$.

We start from the Ohm's law and the continuity equation for the voltage, $U$, and the current density in the channel, $j$

$$
\begin{gathered}
j=-\sigma \frac{\partial U}{\partial x} \\
\frac{\partial \rho}{\partial t}+\frac{\partial j}{\partial x}=0 .
\end{gathered}
$$

Here $\rho$ is the channel charge density, which is proportional to the voltage swing, $U_{0}, U_{0}=U-U_{t h}: \rho=C\left(U-U_{t h}\right)$. The proportionality factor, $C$ is the gate-to-channel capacitance per unit area and $U_{t h}$ is the threshold voltage. Combining Eqs. (1) and (2) we obtain

$$
C \frac{\partial U}{\partial t}-\frac{\partial}{\partial x} \sigma \frac{\partial U}{\partial x}=0
$$

This equation should be solved using the boundary conditions for a long sample

$$
\begin{gathered}
\left.U\right|_{x=0}=V_{g}+U_{a} \cos (\omega t), \text { at the source, and } \\
U \rightarrow \text { const }, \text { for } x \rightarrow \infty, \text { at the drain. }
\end{gathered}
$$


In the above formula, $U_{a}$ is the amplitude of the radiation induced modulation of the source-to-gate voltage and $\omega$ is the angular frequency of the incident $\mathrm{THz}$ radiation. Following the method of Ref. 14, we look for a solution of Eq. (3) as an expansion in powers of $U_{a}$

$$
U=V_{g}+U_{1}+U_{2}
$$

Here $U_{1}(x, t)$ is the $a c$ component proportional to $U_{a}$ and $U_{2}(\mathrm{x})$ is the $d c$ component proportional to $U_{a}^{2}$ (we are not interested in the second harmonic term $\sim U_{a}^{2}$ ). In the first order in $U_{a}$ we obtain

$$
C \frac{\partial U_{1}}{\partial t}-\sigma_{0} \frac{\partial^{2} U_{1}}{\partial x^{2}}=0
$$

where $\sigma_{0}=\sigma\left(V_{g}\right)$. The boundary conditions follow from Eqs. (4) and (5): $U_{1}(0, t)=U_{a} \cos (\omega t), U_{1}(\infty, t)=0$. The solution of this equation is

$$
U_{1}=U_{a} e^{-\kappa x} \cos (\omega t-\kappa x),
$$

where $\kappa^{2}=\frac{\omega C}{2 \sigma_{0}}$ (the above boundary condition described with Eq. (5) is only valid for a long sample, i.e., when $\kappa L \gg$ 1 , where $L$ is the length of the sample).

In the second order in $U_{a}$ after time averaging over the period $2 \pi / \omega$, Eq. (3) takes the form

$$
\frac{\partial}{\partial x}\left[\sigma_{0} \frac{\partial U_{2}}{\partial x}+\left[\frac{d \sigma}{d U}\right]_{U=V_{g}}\left\langle U_{1} \frac{\partial U_{1}}{\partial x}\right\rangle\right]=0
$$

which in particular implies the absence of a $d c$ current. Integrating this equation with the boundary condition $U_{2}(0)=0$ we obtain

$$
U_{2}(x)=\frac{U_{a}^{2}}{4}\left[\frac{1}{\sigma} \frac{d \sigma}{d U}\right]_{U=V_{g}}(1-\exp (-2 \kappa x)) .
$$

Finally, the photoresponse $\Delta U=U_{2}(\infty)-U_{2}(0)$ is given by

$$
\Delta U=\frac{U_{a}^{2}}{4}\left[\frac{1}{\sigma} \frac{d \sigma}{d U}\right]_{U=V_{g}}=\frac{U_{a}^{2}}{4} \frac{d(\ln \sigma)}{d V_{g}} .
$$

If the mobility does not depend on the electron concentration in the channel and the conductivity is proportional to the voltage swing, $U_{0}$, Eq. (11) reproduces the result of Ref. 14 for the non-resonant regime $(\omega \tau \ll 1): \Delta U=U_{a}^{2} /\left(4 U_{0}\right)$, where $\tau$ is the momentum relaxation time.

In general, in a plasma related detection one usually distinguishes four characteristic regimes, which were discussed in more detail in Ref. 11. In the present article, we restrict ourselves to the case of a non-resonant detection regime and a long sample, for which the above model was derived. Our transistors and experimental conditions were chosen so to satisfy these assumptions. Non-resonant detection is the most important case from the point of view of applications, i.e., operation at room temperature and sub-THz frequencies. This is mainly due to the availability of electronic sub- THz sources operating at room temperatures. At lower tempera- tures or higher frequencies of the incoming radiation, one can reach the resonant detection. ${ }^{3,5,7,9}$ Here we limit the frequencies and temperatures to be always in the non-resonant case. Lowering the temperature in which the experiment was carried out was a way to change the channel conductivity (transfer characteristics) and to get an additional verification of the model.

In order to check if the long sample condition, required by the model developed above, is fulfilled in our experiment, we estimate the damping length, $l$, of the plasma perturbation and compare it to the gate length, $L_{g}$. According to Ref. 11, $l=s(\tau / \omega)^{1 / 2}$, where $s=\left(e U_{0} / m_{\text {eff }}\right)^{1 / 2}$ is the plasma wave velocity. When we substitute $s$ in the equation for $l$ we get $l=\left(\mu U_{0} / \omega\right)^{1 / 2}$, where $\mu$ is the electron mobility. To make some estimation we take $U_{0}=0.01 \mathrm{~V}$ (detection signal is maximal close to the threshold voltage), $\mu=0.1 \mathrm{~m}^{2} /(\mathrm{Vs})$ and $\omega=1.8 \mathrm{THz}(\approx 2 \pi \times 300 \mathrm{GHz})$. This gives $l \approx 25 \mathrm{~nm}$, which is far less than $L_{g}$ of the studied transistors (see Table I).

\section{RESULTS AND DISCUSSION}

In Fig. 1, we present the registered photovoltage (PV) signal, $V$, versus the gate voltage for the Si MOSFET N1. Figures 1(a) and 1(b) show, respectively, the amplitude and the phase of the measured signal for different modulation frequencies, $\omega_{m}$, in the absence of the loading resistor, $R_{L}$. Figure 1(c) shows the amplitude of the measured signal with different loading resistances for two modulation frequencies (133 $\mathrm{Hz}$ and $10 \mathrm{kHz})$.

As already mentioned, the registered signal, $V$, is determined by the real photovoltage, $\Delta U$, resulting from the rectification of the $\mathrm{THz}$ radiation (according to the model presented above) and the loading effects that influence the measurement of $\Delta U$. To interpret the loading effects, we use the equivalent circuit of the detector loaded with the measurement setup (cables, connectors, and the preamplifier) as shown in Fig. 2. Then we can write $V$ as

$$
V=\frac{\Delta U}{1+R_{C H} / Z_{L}},
$$

where $R_{C H}$ is the channel resistance and $Z_{L}$ is the complex load impedance of the setup determined by the relation

$$
\frac{1}{Z_{L}}=\frac{1}{R_{I N}}+\frac{1}{R_{L}}+i \omega_{m} C_{L} .
$$

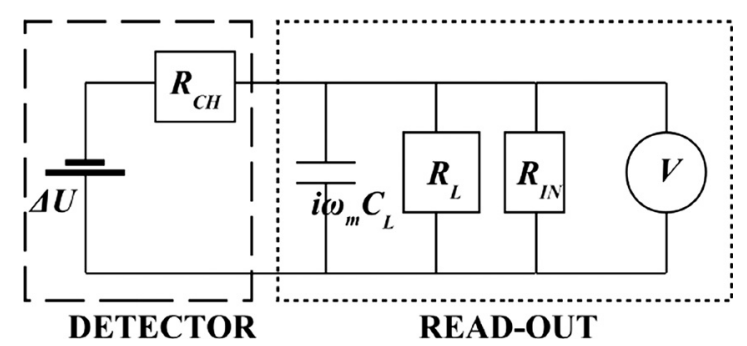

FIG. 2. Equivalent electrical circuit used to understand the loading effects. Here $V$ is the measured photovoltage, $\Delta U$ is the photoresponse of the transistor, $R_{C H}$ is the channel resistance, $C_{L}$ is a parasitic capacitance, $\omega_{m}$ is the modulation frequency, $R_{L}$ is the external load resistor (if present), and $R_{I N}$ is the input resistance of the preamplifier. 
Here $R_{I N}$ is the input resistance of the preamplifier, $R_{L}$ is an external load resistor, and $C_{L}$ represents the parasitic capacitance of the measurement set-up. The inductance component contribution to the load impedance was omitted, as in our case it was negligibly small because of low modulation frequencies.

From Fig. 1, one can see that with the decrease of the gate voltage, the amplitude of the registered signal achieves its maximum and then drops down. This can be easily understood by analyzing Eq. (12). At high gate voltages, $V_{g}-U_{t h}>0$, the channel resistance is small, the loading effect is negligible, and the measured voltage signal $V \approx \Delta U$ does not depend on $Z_{L}$. It means that for large $V_{g}$, all the detection curves tend to coincide (see Fig. 1(a)). At small gate voltages, $V_{g}-U_{t h}<0$, the transistor is almost closed and the channel resistance diverges. In this case, the measured voltage signal takes the form of $V \approx \Delta U Z_{L} / R_{C H}$ and decays exponentially to zero. The maximum of the voltage signal amplitude is reached at the gate voltage such that $R_{C H}\left(V_{g}\right) \sim\left|Z_{L}\right|$. Alternating the modulation frequency (Fig. 1(a)) or the loading resistance (Fig. 1(c)), we change the loading impedance (Eq. (13)) and the position of the maximum shifts. The evolution of the signal phase (Fig. 1(b)) can also be explained using Eqs. (12) and (13). At large $V_{g}$, we have $V \approx \Delta U$ and the signal phase is zero. Then for lower gate voltages, the phase is dominated by the ratio $1 /\left(1+R_{C H} / Z_{L}\right)$ and finally, when the transistor is closed, the signal phase equals to the phase of $Z_{L}$. For high modulation frequencies $Z_{L} \approx 1 /\left(\omega_{m} C_{L}\right)$ and the phase of $Z_{L}$ equals $-\pi / 2$. For smaller frequencies, $1 /\left(i \omega_{m} C_{L}\right)$ becomes comparable with the input resistance of the preamplifier and the phase of $Z_{L}$ is determined by the ratio between them.

We measured the $d c$ transfer characteristics (Fig. 3) of the FET in the absence of the $\mathrm{THz}$ radiation for small source-to-drain voltage $(1 \mathrm{mV})$ to experimentally establish the relation between the non-resonant $\mathrm{THz}$ photoresponse and the channel conductivity. In this situation, the photoresponse (Eq. (11)) takes the form

$$
\Delta U=\frac{U_{a}^{2}}{4} \frac{d}{d V_{g}} \ln \left[I_{D S}\left(V_{g}\right)\right]
$$

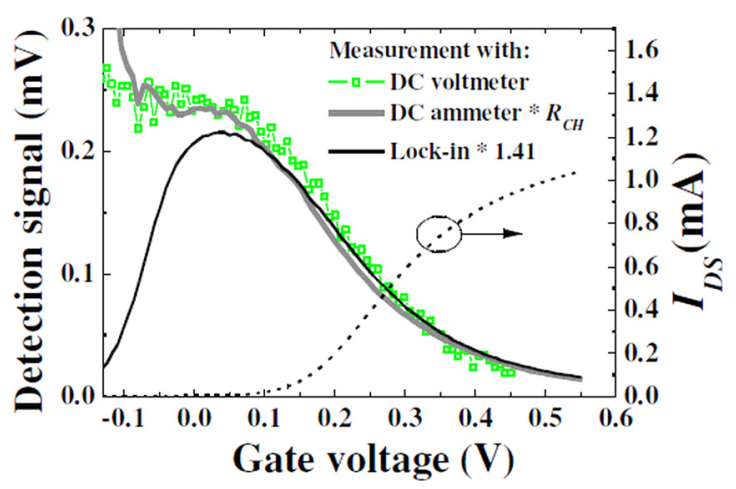

FIG. 3. (Color online) Comparison of the detection signal of Si MOSFET $\mathrm{N} 1$ measured at $R_{L}=0$ with different instruments: $d c$ voltmeter (squares), $d c$ ammeter multiplied by the channel resistance (thick gray line), and lock-in at $\omega_{m}=133 \mathrm{~Hz}$ multiplied by $\sqrt{2}$ (thin black line). Right scale (dashed line): $d c$ transfer characteristics for $1 \mathrm{mV}$ of source-to-drain voltage. where $I_{D S}\left(V_{g}\right)$ is the $d c$ source-to-drain current dependence on the gate voltage ( $d c$ transfer characteristic). Taking into account the loading we find the final expression describing the measured photovoltage

$$
V=\frac{A}{1+R_{C H} / Z_{L}} \frac{d}{d V_{g}} \ln \left[I_{D S}\left(V_{g}\right)\right] .
$$

Here we denoted the unknown $U_{a}^{2} / 4$ scaling factor by $A$, which is the only normalization parameter in our analysis. Physically $A$ is determined by the coupling efficiency of the incoming radiation to the antenna together with a certain impedance of the transistor. As we checked, $A$ is proportional to the power of incoming radiation, but a precise determination of this factor, especially at $\mathrm{THz}$ frequencies, is still an unsolved problem. When fitting the maximum of the calculated photoresponse (using Eq. (15)) to the experimentally measured curve for the Si MOSFET N1, we got the same value of $A$ for all the curves presented in Fig. 1, $A=1.25 \times 10^{-4} \mathrm{~V}^{2}$. All other parameters were determined experimentally and they were not fitting parameters. One universal factor $A$ shows, according to our model, that the response signal, $\Delta U$, stays the same (the coupling condition does not change in particular) and the loading impedance influences just the measured voltage response. The calculated photoresponse curves ( $V$ calculated in accordance with Eq. (15)) are shown in Fig. 1 as black solid lines. The thick gray solid line in Fig. 1(a) shows $\Delta U$ calculated from Eq. (14) and corresponds to an infinite loading impedance $\left(Z_{L} \rightarrow\right.$ $\infty)$. As one can see, the measured photovoltage (points) and the one calculated using the channel conductivity obtained from the $d c$ transfer characteristic (lines) are in a very good agreement both in amplitude and phase for all modulation frequencies and loading resistances.

The validity of our approach and the role of the loading effects were independently confirmed by measurements done with a $d c$ voltmeter and a $d c$ ammeter characterized by a very high (of the order of $\mathrm{T} \Omega$ ) and a very low input impedance, respectively. Photoresponse measurements done in a different way are compared in Fig. 3. The voltage signal measured with a lock-in amplifier is drawn as a thin black line and the one measured with a $d c$ voltmeter as a line with squares. The lock-in signal is multiplied by $\sqrt{2}$ to take into account that the lock-in measures the effective voltage. It is enough because the excitation had a sinusoidal character due to a large diameter of the incident beam, of the order of an opening in the chopper. No other rescaling was used in Fig. 3 . For the $d c$ measurements, the chopper was turned on so to have the same average radiation intensity as for the $a c$ measurements with the lock-in. The third detection curve (thick gray line) was measured with the $d c$ ammeter and then multiplied by $R_{C H}$ obtained from the $d c$ transfer characteristics (dashed line, right scale). For the high gate voltages, all three curves coincide. In the case of the $a c$ measurements for the lower gate voltages, we can see that the voltage drops near the threshold voltage, whereas for both $d c$ curves, the photovoltage reaches a plateau, as predicted by Eq. (15) in the case of a very high $Z_{L}$ (see the thick gray curve in Fig. 1(a)). This is due to the fact that in $d c$ measurements one can 
neglect the capacitive load of the read-out circuit and the impedance of the $d c$ instruments is much bigger than this of the preamplifier used in the $a c$ lock-in measurements.

The presented results show that using our model and taking into account the loading effects, one can fully describe the experimental results for the investigated $\mathrm{Si}$ MOSFET N1 (Fig. 1). Furthermore, we checked the validity of the model in case of $\mathrm{THz}$ detection for various operating temperatures for III-V HEMTs (GaAs, GaN) and for an additional silicon MOSFET marked by N2. The results are presented in Figs. 4-6. Figures 4(a), 5(a), and 6(a) show the $d c$ transfer characteristics and points in Figs. 4(b), 5(b), and 6 (b) are the experimental photovoltage results. No additional external loading resistor was used. One can see that with the temperature decrease, the threshold voltage shifts to higher gate voltages and the slope of the respective transfer characteristic becomes steeper. This happens due to a decrease of the carrier density and an increase of the carrier mobility, respectively. The maxima of the photovoltage shift towards higher gate voltages in the same way as the threshold voltage does. The solid lines in Figs. 4(b), 5(b), and 6(b) represent the photovoltage signal calculated from Eq. (15) with the normalization parameter $A$. In all cases, the calculated signal is very close to the measured photovoltage.

In the case of the broadband non-resonant detection, the rectification of the $\mathrm{THz}$ radiation by $\mathrm{FETs}$ was also explained by the so called resistive self-mixing. ${ }^{16}$ However in the case of transistors with the gate longer than the plasma damping length, the distribution of the charge density perturbation along the channel is not linear. In this case the strict resistive self-mixing approach is not valid. More detailed

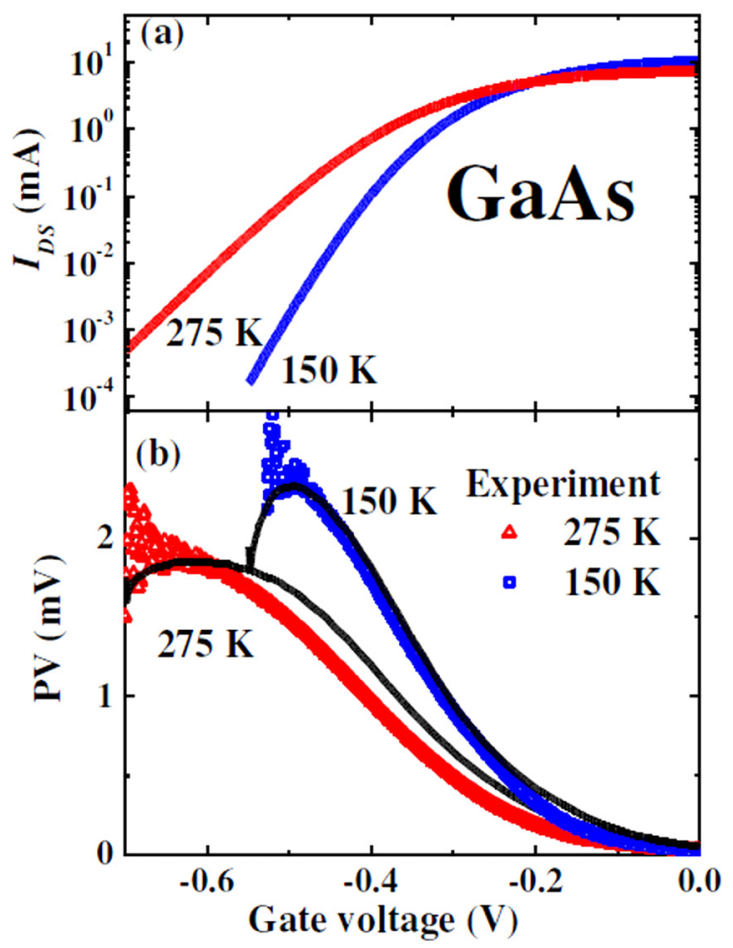

FIG. 4. (Color online) GaAs HEMT: (a) $d c$ transfer characteristics, $I_{D S}\left(V_{g}\right)$ and (b) measured (points) and the calculated (Eq. (15), lines) photovoltages at two temperatures: $275 \mathrm{~K}$ and $150 \mathrm{~K}$ with the parameter $A=7 \times 10^{-5} \mathrm{~V}^{2}$ and $5.2 \times 10^{-5} \mathrm{~V}^{2}$, respectively.

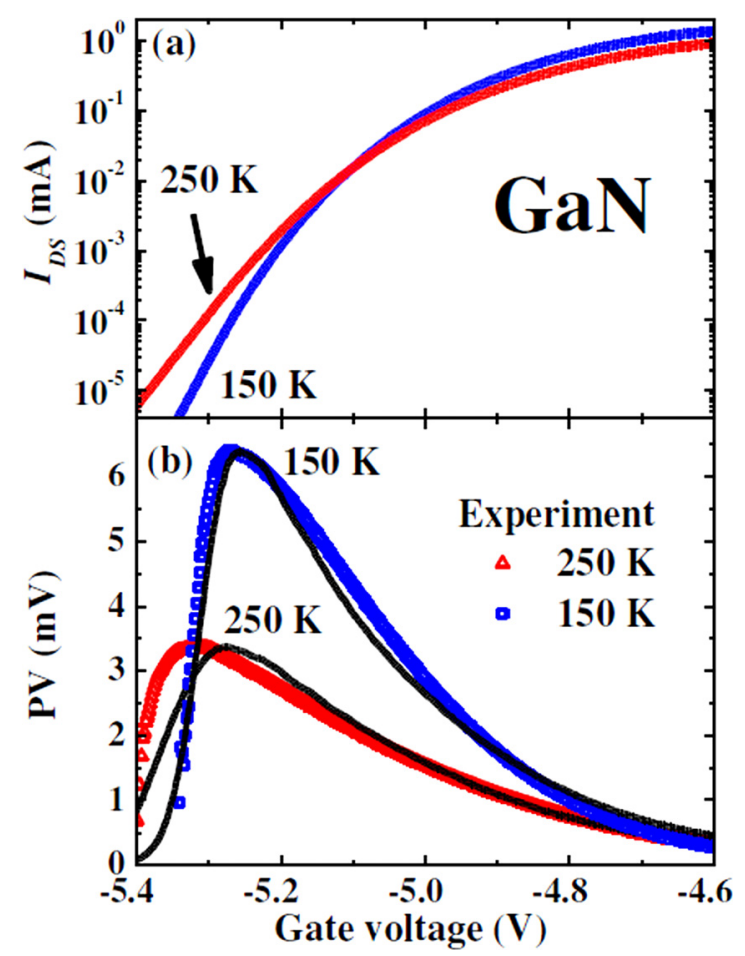

FIG. 5. (Color online) GaN HEMT: (a) $d c$ transfer characteristics, $I_{D S}\left(V_{g}\right)$ and (b) measured (points) and the calculated (Eq. (15), lines) photovoltages at two temperatures: $250 \mathrm{~K}$ and $150 \mathrm{~K}$ with the parameter $A=1.24 \times 10^{-4}$ $\mathrm{V}^{2}$ and $1.84 \times 10^{-4} \mathrm{~V}^{2}$, respectively.

discussion of the electronic and physics related approaches to $\mathrm{THz}$ rectification can be found in Ref. 11. Only recently the developed so called distributed self-mixing model ${ }^{16-18}$ can be used for numerical simulation of the $\mathrm{THz}$ rectification

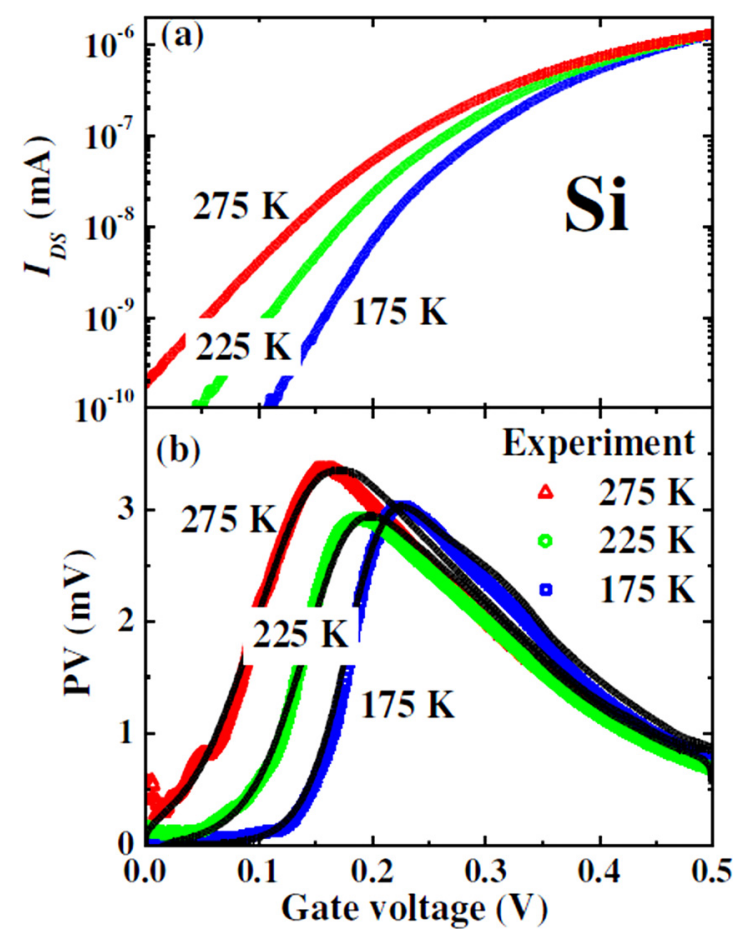

FIG. 6. (Color online) Si MOSFET N2: (a) $d c$ transfer characteristics, $I_{D S}\left(V_{g}\right)$ and (b) measured (points) and the calculated (Eq. (15), lines) photovoltages at three temperatures: $275 \mathrm{~K}, 225 \mathrm{~K}$, and $175 \mathrm{~K}$ with the parameter $A=1.41 \times 10^{-2} \mathrm{~V}^{2}, 1.08 \times 10^{-2} \mathrm{~V}^{2}$, and $1.03 \times 10^{-2} \mathrm{~V}^{2}$, respectively. 
in FETs. Therefore, it is worth to stress that the theoretical model presented here allows to predict the photoresponse analytically - using as input information the easily measurable or calculable static transfer characteristics.

\section{CONCLUSIONS}

We would like to emphasize that our approach gives a simple way to calculate the $\mathrm{THz}$ photoresponse not only close to the maximal signal value but also in the whole range of the gate voltage. It is worth to note that a very good agreement between the measured and the calculated non-resonant $\mathrm{THz}$ photovoltages was obtained also in the subthreshold region, where the channel resistance grows exponentially and the loading effects start to play an important role. The successful interpretation of the results was possible because of two new developments: (i) the theoretical model that relates the $\mathrm{THz}$ responsivity to the change of the total conductivity and not only to the change of the carrier density, that eliminates necessity of a constant mobility assumption and (ii) taking into account not only resistive but also capacitive part of the loading impedance. This capacitive load is especially important in the subthreshold range because the high channel resistance leads to the important $R C$ effects even at low modulation frequencies.

With a proper design of a high input resistance preamplifier and a readout circuit, one can avoid the parasitic impedances and related loading effects in the case of a focal plane array where a FET in each pixel works as $\mathrm{THz}$ detector. The $\mathrm{THz}$ responsivity characteristics can be easily obtained, especially in the case of the mature CMOS technology, in which the gate leakages are negligible and it is possible to design the $d c$ transfer characteristics even before the manufacturing stage. The approach described above will also help in designing new low cost $\mathrm{Si}-\mathrm{CMOS}$ based focal plane arrays for future $\mathrm{THz}$ imaging systems.

In summary, in this work, we find a relation between the non-resonant $\mathrm{THz}$ photoresponse and the $d c$ channel conductivity of FETs. The proposed model is based on the Dyakonov-Shur theory which describes $\mathrm{THz}$ detection as a rectification of overdamped plasma waves excited in the FET channel by the incoming $\mathrm{THz}$ radiation. Our model allows to calculate the photovoltage dependence on the gate voltage from the $d c$ transfer characteristics that can be easily measured or simulated at the stage of a transistor design. The theoretical model completed by the loading effects gives a very good description of the experimental results. Therefore the approach described here gives the prospects for designing new, FET-based focal plane arrays for the future THz imaging systems.

\section{ACKNOWLEDGMENTS}

We acknowledge M. I. Dyakonov for many helpful discussions concerning the theoretical part of the work. We also thank III-V Lab Marcoussis and IEMN-LILLE for providing the GaN FETs and CEA-LETI and ST Microelectronics for providing the Si-MOSFETs. This work is supported by GISTERALAB Montpellier, GDR2987, GDR-I THz, FranceJapan Thz project WITH and the Russian Ministry of Education and Science (SC 14.740.11.0892). We also thank the French Embassy in Moscow for a substantial support in establishing the Russian-French collaboration in the field of THz science and technology.

${ }^{1}$ J.-Q. Lu, M. S. Shur, J. L. Hasler, L. Sun, and R. Weikle, IEEE Electron Device Lett. 19, 373 (1998).

${ }^{2}$ J.-Q. Lu and M. S. Shur, Appl. Phys. Lett. 78, 2587 (2001).

${ }^{3}$ W. Knap, Y. Deng, S. Rumyantsev, and M. S. Shur, Appl. Phys. Lett. 81, 4637 (2002).

${ }^{4}$ W. Knap, V. Kachorovskii, Y. Deng, S. Rumyantsev, J.-Q. Lu, R. Gaska, M. S. Shur, G. Simin, X. Hu, M. Asif Khan, C. A. Saylor, and L. C. Brunel, J. Appl. Phys. 91, 9346 (2002).

${ }^{5}$ W. Knap, Y. Deng, S. Rumyantsev, J.-Q. Lu, M. S. Shur, C. A. Saylor, and L. C. Brunel, Appl. Phys. Lett. 80, 3433 (2002).

${ }^{6}$ W. Knap, F. Teppe, Y. Meziani, N. Dyakonova, J. Łusakowski, F. Boeuf, T. Skotnicki, D. Maude, S. Rumyantsev, and M.S. Shur, Appl. Phys. Lett. 85, 675 (2004).

${ }^{7}$ F. Teppe, W. Knap, D. Veksler, M. S. Shur, A. P. Dmitriev, V. Yu. Kachorovskii, and S. Rumyantsev, Appl. Phys. Lett. 87, 052107 (2005).

${ }^{8}$ R. Tauk, F. Teppe, S. Boubanga, D. Coquillat, W. Knap, Y. M. Meziani, C. Gallon, F. Boeuf, T. Skotnicki, C. Fenouillet-Beranger, D. K. Maude, S. Rumyantsev, and M. S. Shur, Appl. Phys. Lett. 89, 253511 (2006).

${ }^{9}$ A. El Fatimy, F. Teppe, N. Dyakonova, W. Knap, D. Seliuta, G. Valušis, A. Shchepetov, Y. Roelens, S. Bollaert, A. Cappy, and S. Rumyantsev, Appl. Phys. Lett. 89, 131926 (2006).

${ }^{10}$ A. El Fatimy, N. Dyakonova, F. Teppe, W. Knap, N. Pala, R. Gaska, Q. Fareed, X. Hu, D. B. Veksler, S. Rumyantsev, M. S. Shur, D. Seliuta, G. Valusis, S. Bollaert, A. Shchepetov, Y. Roelens, C. Gaquiere, D. Theron, and A. Cappy, Electron. Lett. 42, 1342 (2006).

${ }^{11}$ W. Knap, M. Dyakonov, D. Coquillat, F. Teppe, N. Dyakonova, J. Lusakowski, K. Karpierz, M. Sakowicz, G. Valušis, D. Seliuta, I. Kašalynas, A. El Fatimy, Y. M. Meziani, and T. Otsuji, J. Infrared Millim. Terahz. Waves 30, 1319 (2009).

${ }^{12}$ W. Knap, H. Videlier, S. Nadar, D. Coquillat, N. Dyakonova, F. Teppe, M. Bialek, M. Grynberg, K. Karpierz, J. Lusakowski, K. Nogajewski, D. Seliuta, I. Kašalynas, and G. Valušis, Opto-Electron. Rev. 18, 225 (2010).

${ }^{13}$ M. Dyakonov and M. Shur, Phys. Rev. Lett. 71, 2465 (1993).

${ }^{14}$ M. Dyakonov and M. Shur, IEEE Trans. Electron Devices 43, 380 (1996).

${ }^{15}$ A. Lisauskas, W. von Spiegel, S. Boubanga-Tombet, A. El Fatimy, D. Coquillat, F. Teppe, N. Dyakonova, W. Knap, and H. G. Roskos, Electron. Lett. 44, 408 (2008).

${ }^{16}$ A. Lisauskas, U. Pfeiffer, E. Öjefors, P. H. Bolìvar, D. Glaab, and H. G. Roskos, J. Appl. Phys. 105, 114511 (2009).

${ }^{17}$ E. Öjefors, U. R. Pfeiffer, A. Lisauskas, and H. G. Roskos, IEEE J. SolidState Circuits 44, 1968 (2009).

${ }^{18}$ E. Ojefors, N. Baktash, Y. Zhao, and U. Pfeiffer, "Terahertz imaging detectors in a 65-nm CMOS SOI technology," in 36th European SolidState Circuits Conference (Sevilla, Spain, 2010).

${ }^{19}$ W. Stillman, M. S. Shur, D. Veksler, S. Rumyantsev, and F. Guarin, Electron. Lett. 43, 422 (2007).

${ }^{20}$ A. El Fatimy, N. Dyakonova, Y. Meziani, T. Otsuji, W. Knap, S. Vandenbrouk, K. Madjour, D. Théron, C. Gaquiere, M. A. Poisson, S. Delage, P. Prystawko, and C. Skierbiszewski, J. Appl. Phys. 107, 024504 (2010).

${ }^{21}$ S. Nadar, H. Videlier, D. Coquillat, F. Teppe, M. Sakowicz, N. Dyakonova, W. Knap, D. Seliuta, I. Kašalynas, and G. Valušis, J. Appl. Phys. 108, 054508 (2010).

${ }^{22}$ F. Schuster, D. Coquillat, H. Videlier, M. Sakowicz, F. Teppe, L. Dussopt, B. Giffard, T. Skotnicki, and W. Knap, Opt. Express 19, 7827 (2011). 